men. Im Rahmen der Störungen des Magen-Darmtrakts ist die diabetische Diarrhö besonders lästig.

\section{Unerklärliche Durchfälle}

Unerklärliche Durchfälle treten besonders nachts oder nach Mahlzeiten auf. Auch das Urogenitalsystem kann betroffen sein. Der fehlende Dehnungsreiz führt zur Überfüllung der Blase und schließlich zur Überlaufblase, bei der spontan größere Urinmengen abfließen. Potenzstörungen sind durch autonome Funktionsstörungen erklärbar, die zu Störungen der Erektion führen.

Bei der Small fiber neuropathy sind vorwiegend die distalsten (sensiblen) Nervenendigungen betroffen. Die Patienten klagen über schmerzhafte Missempfindungen. Bei der Untersuchung lassen sich Ausfälle kaum objektivieren.

Die Mononeuropathien sind viel seltener. Ausfälle einzelner Nerven, Wurzeln oder Plexus können akut auftreten,

und im Gegensatz zur symmetrischen Polyneuropathie zei-

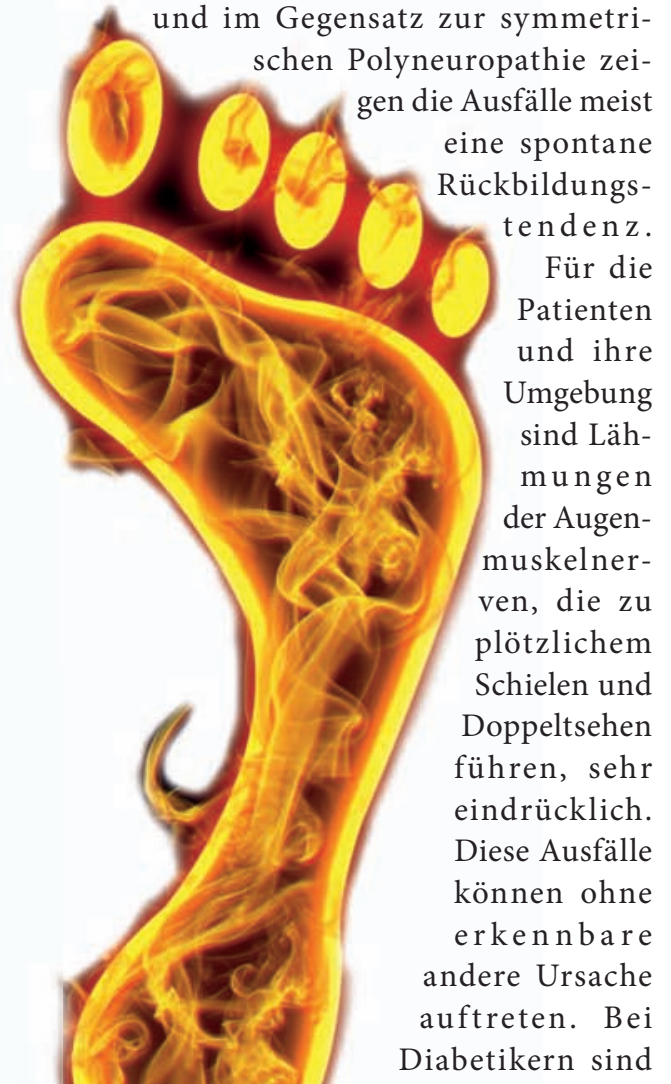

aber auch Kompressionsneuropathien gehäuft (z. B. Karpaltunnelsyndrom).

\section{Plötzlich kraftlos in den Oberschenkeln?}

Eine Sonderform ist die sog. «diabetische Amyotrophie». Eine plötzlich auftretende Kraftlosigkeit in den Muskeln des Oberschenkels ist von heftigen neuralgischen Schmerzen in diesen Muskeln begleitet. Vielfach ist das Symptombild ohne weitere Abklärungen nur schwer von einem lumbalen Bandscheibenvorfall abzugrenzen.

Beim Erheben der Anamnese und bei der neurologischen Untersuchung ist ein besonderes Augenmerk auf mögliche autonome Störungen zu legen, die von den Patienten meist nicht spontan erwähnt werden. Zur Bestätigung der Diagnose dienen in erster Linie elektrophysiologische Untersuchungen (Elektromyo- und Elektroneurografie). Wertvoll können auch autonome Funktionstests sein, zum Beispiel Schweisstest oder Variabilität des RR-Intervalls. Eine Nerven- oder Muskelbiopsie ist in der Regel nicht angezeigt.

Polyneuropathien können zahlreiche verschiedene Ursachen haben. Mit den bisher erwähnten diagnostischen Methoden kann über die Ursache noch nichts Sicheres gesagt werden. Für die Sicherung der Diagnose einer diabetischen Neuropathie muss einerseits eine Störung des Zuckerstoffwechsels nachgewiesen werden, andererseits müssen weitere mögliche Ursachen ausgeschlossen werden. Dazu gehören andere Stoffwechselstörungen, entzündliche Erkrankungen, Kontakte mit toxischen Substanzen, übermäßiger Alkoholkonsum, Mangelernährung und erbliche Krankheitsformen.

\section{Füße und Risikofaktoren beachten}

Trotz großer Anstrengungen konnte in den letzten Jahrzehnten kein Durchbruch in der Behandlung erzielt werden. Eine kurative Therapie ist nicht in Sicht. Einzig bei der Schmerzbekämpfung $\rightarrow$

\section{Propofol lieber in die Armbeuge spritzen}

Startet eine Narkose mit Propofol, ist der Schmerz dieser Injektion - ausgelöst durch den Kontakt des Hypnotikums mit der Venenwand - oft das erste, woran sich die Patienten nach der Op erinnern. Verhindert werden kann dies, indem der Wirkstoff nicht in den Handrücken, sondern in die Venen in der Armbeuge gespritzt wird. Zu dieser Erkenntnis gelangten die Autoren einer Meta-Analyse mit 25.260 Patienten. Grund sei das größere Lumen und der schneller Durchfluss in den Armvenen. Jalota L et al, BMJ 2011, 342:d1110

\section{Besser schlafen mit weniger Opioiden}

Die nur einmal tägliche Einnahme eines Opioids ist mit vergleichsweise wenig Schlafproblemen behaftet. In der internationalen Patientenumfrage PARES wurden 875 Patienten zu ihrer Schmerztherapie befragt. Bei dreimal täglicher Medikamenteneinnahme berichteten $43 \%$ über Einschlafprobleme, unter ein- oder zweimal täglicher Einnahme nur ein Fünftel. Ein Drittel der Patienten mit Einmaldosierung hatte gar keine Einschlafprobleme. 22. Dt. Schmerz und Palliativkongress 2011

\section{Selbst ist der geplagte Rücken}

Am Arbeitsplatz ergreifen Patienten mit Rückenschmerz oft selbst die Initiative: Sie versuchen, die Dauer der Werktätigkeit einzugrenzen und sich bestimmter Aufgaben zu entledigen. Professionelle Unterstützung bei der Gestaltung der Arbeit suchen die meisten nicht, so eine Studie. Arbeitsmediziner werden am ehesten nach längerer Krankschreibung einbezogen. Viele zeigen zudem übervorsichtige Verhaltensweisen, aus Angst, der Arbeit nicht mehr gewachsen zu sein. BMC Musculoskelet Disord 2010, 11:277 